\title{
Procedure Agents Completion Status
}

National Cancer Institute

\section{Source}

National Cancer Institute. Procedure Agents Completion Status. NCI Thesaurus. Code C162336.

A term used to describe the state or condition of the completeness of the procedure agent administration data. 